Radial and Nonradial Pulsations as Probes of Stellar Physics

ASP Conference Series, Vol. 259, 2002

C. Aerts, T.R. Bedding, \& J. Christensen-Dalsgaard, eds.

\title{
Do Temperature Variations at the Surface of a Hot Non-Radial Pulsator Change Significantly the Line-Profile Variations?
}

\author{
J. De Ridder, C. Aerts
}

Instituut voor Sterrenkunde, Katholieke Universiteit Leuven, Belgium

\author{
M.-A. Dupret, C. Neuforge \\ Institut d'Astrophysique et de Géophysique, Université de Liège, \\ Belgium
}

\begin{abstract}
We present simulations of line-profile variations of Silicon lines in slowly-rotating non-radially pulsating $\beta$ Cephei stars and SPB stars. Our goal is to examine how sensitive Si lines are to the nonadiabatic temperature variations at the surface of the star. We find that for realistic amplitudes of the velocity field, and for realistic amplitudes and phase differences for the non-adiabatic temperature and gravity variations, the Silicon lines variations are very little affected.
\end{abstract}

\section{Introduction}

Many of the $\beta$ Cephei stars and slowly pulsating B (SPB) stars show line-profile variations. One can expect that these line-profile variations contain a lot of information about the pulsation modes. It is therefore important and interesting to study them and to understand them well. The problem we tackled is the following. For quite some time, since Osaki (1971), it is well known that lineprofile variations of $\beta$ Cephei stars and SPB stars can be well mimicked with a non-radial velocity field. We also know, however, that at the surface of the star, not only the velocity changes with time, but also for example, the temperature. This can be explicitely seen in the observational data, for example in the photometrically determined effective temperature variations, or in the equivalent width (EW) variations of spectral lines. A relevant question is: what is the influence of the local non-adiabatic temperature variations on the line-profile variations? It may cause $\mathrm{EW}$ variations, but even for small $\mathrm{EW}$ variations the line shape may still be significantly affected. For rapidly rotating pulsating stars, claims have been made in the literature (e.g. Balona, 1987), that temperature effects may even overshadow the velocity effects on the line profile. For slowly rotating pulsating stars - the ones we study here - the effect is still unclear, partially because of the uncertain amplitude of the non-adiabatic temperature variations. If the effect is large, then ignoring temperature variations may jeopardize a mode identification done for example with line-profile fitting or with the moment method. We therefore modelled line-profile variations of Silicon lines (which are quite popular to study B stars, e.g. Uytterhoeven et al., 2001, and 
De Cat et al., 2000), and checked how sensitive these lines are to temperature variations.

\section{Line profile modelling}

We implemented a line-profile modelling code, called PULSTAR, and in what follows, we give an overview of its basic ingredients.

- As is usually done, we adopt the one-layer approximation. This means that we assume that the spectral line is formed in one layer only. The question is of course: which layer ? We chose the layer that contributes most to the line depression. We therefore computed line contribution functions for all our Silicon lines, with the formalism described in Albrow \& Cottrell (1996). Doing so, we learned for the first time where in the photosphere these Silicon lines are actually formed, and this turned out to be always quite high in the photosphere, for example at $\log \tau_{\text {ross }}=-2$ for the SiII doublet around $413 \mathrm{~nm}$, in SPB stars.

- There is no restriction on the number of pulsation modes, but currently each mode is described by one spherical harmonic $Y_{\ell}^{m}$ with degree $\ell$ and azimuthal number $m$.

- As the surface is distorted due to the non-radial displacement field, we computed the local surface normal in each point of the surface.

- The emergent intensity $I_{\lambda}(\mu)$ was computed by interpolation in large precomputed Kurucz intensity grids. These intensity grids depend on the wavelength $\lambda$, on $\mu$ which is the cosine of the angle between the local surface normal and the line of sight, on the effective temperature $T_{\text {eff }}$, and on the gravity $g$. We take into account that for a non-radially pulsating star, $\mu, T_{\text {eff }}$, and $g$ vary over the stellar surface and with time.

The line-profile code divides the stellar surface in many small cells, and computes for each cell the pulsational velocity field, the local surface normal, and the local (Doppler-shifted) intensity spectrum. It then integrates over the entire visible stellar surface to obtain the observed flux spectrum. Doing this for several phases during the pulsation cycle, it returns a time series of spectra.

A very important part of line-profile modelling, is to provide reliable input parameters for PULSTAR. In particular, care should be taken to adopt realistic values for the amplitude of the pulsational velocity field, and for the amplitude and non-adiabatic phase difference of the local $T_{\text {eff }}$ variation and the local $g$ variation. In particular, the local $T_{\text {eff }}$ variations are difficult to estimate, because from the base of the partial ionization zones to the outermost layers of the atmosphere - where Si-lines are formed - the pulsation is no longer adiabatic, but becomes highly non-adiabatic. We therefore adapted our non-adiabatic pulsation code to improve the computations of the non-adiabatic eigenfunctions in the atmosphere. The basic ideas behind our approach to do so, are briefly given in the next section. 


\section{Non-adiabatic temperature variations in the atmosphere}

Because in a non-radially pulsating star, all eigenfunctions have an angular dependence, it is useful to use the notion of a "local atmosphere" which is the gas column at a given angular position $(\theta, \varphi)$. An important point is that the diffusion approximation as well as the Eddington approximation do not give the correct temperature distribution in the outer atmosphere of a star. A much more accurate temperature distribution can be obtain from the non-grey Kurucz atmosphere models for example. It is such models that we use in our method.

Given the chemical composition, the equilibrium temperature of a Kurucz atmosphere model can be written as a function of the Rosseland mean optical depth $\tau, T_{\text {eff }}$ and $g$ :

$$
T=T\left(\tau, T_{\text {eff }}, \log g\right) .
$$

Our main approximation is to assume that, at each phase during the pulsation cycle, the $T(\tau)$ law in the local atmosphere is the same as the $T(\tau)$ law of an equilibrium Kurucz atmosphere model. Of course, during the pulsation, the two parameters $T_{\text {eff }}$ and $g$ characterising the equilibrium atmosphere model, and thus the local atmosphere, are varying with time and are function of $\theta$ and $\varphi$. This approximation can be supported by the fact that the thermal relaxation time of the atmosphere $\left(\tau_{\text {th }} \approx 2 s\right.$ for a typical $\beta$ Cephei) is very short compared to the typical pulsation periods, so that it can be assumed in a very good approximation, that the local atmosphere remains in radiative equilibrium during the pulsation. Subsequently, we perturb relation(1):

$$
\frac{\delta T}{T}=\frac{\partial \ln T}{\partial \ln T_{\mathrm{eff}}} \frac{\delta T_{\mathrm{eff}}}{T_{\mathrm{eff}}}+\frac{\partial \ln T}{\partial \ln g} \frac{\delta g}{g}+\frac{\partial \ln T}{\partial \ln \tau} \frac{\delta \tau}{\tau}
$$

and use it to replace the energy and diffusion equation in the atmosphere together with appropriate boundary and matching conditions. So our nonadiabatic pulsation code still uses the diffusion approximation in the interior but it uses Exp.(1) in the atmosphere. Finally, another particularity of our non-adiabatic code is the special care given to the modelling of the radiative pressure in the atmosphere, avoiding to use the law $P_{R}=(1 / 3) a T^{4}$, which is no longer valid in the outermost layers of the atmosphere.

We emphasize that we neglected the interaction between rotation and pulsation, so the non-adiabatic pulsation code does not apply to rapidly rotating stars. Therefore, for the simulations presented here, we restrict ourselves to slowly-rotating stars.

\section{Results}

Now that we have more reliable values for the amplitude and non-adiabatic phase difference of both the $T_{\text {eff-variation and the } g \text {-variation, we computed }}$ spectral line time series for many modes for a $\beta$ Cephei star model as well as for an SPB star model. We compared spectral lines computed with and without non-adiabatic temperature variations. Our results can be summarized as follows:

- We find that line-profile variations of Silicon lines are very little affected by temperature variations at the surface of the star. 
- We find relative EW variations up to a few percent for $\ell=1$ and $\ell=2$ modes. For higher degree modes, the EW variations are so small that they would be very difficult to measure.

- The extra non-adiabatic phase shift of the $T_{\text {eff }}$ variation can be up to 40 degrees, and this is reflected in the phase difference between the EW and the radial velocity. That is, the EW does not necessarily reach its maximum exactly when the observed radial velocity is zero.

The general conclusion we can make, is that for slowly-rotating non-radially pulsating B stars, the line-profile variations of Silicon lines can be well approximated with a pulsational velocity field only.

The results of this research will be published in detail in Dupret et al. (2002), and in De Ridder et al. (2002).

\section{References}

Albrow, M.D. \& Cottrell, P.L. 1996, MNRAS, 278, 337

Balona, L.A. 1987, MNRAS, 224, 41

De Cat, P., Aerts, C., De Ridder, J., Kolenberg, K., Meeus, G., \& Decin, L. 2000, A\&A, 355, 1015

De Ridder, J., Dupret, M.-A., Neuforge, C., \& Aerts C. 2002, A\&A, in press

Dupret, M.-A., De Ridder, J., Neuforge, C., Aerts, C., \& Scuflaire, R. 2002, $A \& A$, in press

Osaki, Y. 1971, PASJ, 23485

Uytterhoeven, K., Aerts, C., De Cat, P., De Mey, K., Telting, J.H., Schrijvers, C., De Ridder, J., Daems, K., Meeus, G., \& Waelkens, C. 2001, A\&A, 371,1035

\section{Discussion}

S. Owocki : You mention that you include the effect of the normal variation of your local atmosphere. How much does the normal deviate from the local radial vector?

J. De Ridder : For the realistic amplitudes we chose, the deviation of the local surface normal from the local radial vector was never more than a few degrees. 